OPEN ACCESS

Edited by:

Michael Thomas-Poulsen,

University of Copenhagen, Denmark

Reviewed by:

Laura Steindler,

University of Haifa, Israel

Robert W. Thacker,

Stony Brook University, USA

${ }^{*}$ Correspondence:

Zhiyong L

zyli@sjtu.edu.cn

Specialty section:

This article was submitted to

Microbial Symbioses,

a section of the journa

Frontiers in Microbiology

Received: 30 April 2016 Accepted: 12 August 2016

Published: 25 August 2016

Citation

Liu F, Li J, Feng G and Li Z (2016) New Genomic Insights into

"Entotheonella" Symbionts in Theonella swinhoei: Mixotrophy, Anaerobic Adaptation, Resilience,

and Interaction

Front. Microbiol. 7:1333.

doi: 10.3389/fmicb.2016.01333

\section{New Genomic Insights into "Entotheonella" Symbionts in Theonella swinhoei: Mixotrophy, Anaerobic Adaptation, Resilience, and Interaction}

\author{
Fang Liu, Jinlong Li, Guofang Feng and Zhiyong Li* \\ Marine Biotechnology Laboratory, Department of Bioengineering, State Key Laboratory of Microbial Metabolism-School of \\ Life Sciences and Biotechnology, Shanghai Jiao Tong University, Shanghai, China
}

"Entotheonella" (phylum "Tectomicrobia") is a filamentous symbiont that produces almost all known bioactive compounds derived from the Lithistida sponge Theonella swinhoei. In contrast to the comprehensive knowledge of its secondary metabolism, knowledge of its lifestyle, resilience, and interaction with the sponge host and other symbionts remains rudimentary. In this study, we obtained two "Entotheonella" genomes from T. swinhoei from the South China Sea through metagenome binning, and used a RASTtk pipeline to achieve better genome annotation. The high average nucleotide index values suggested they were the same phylotypes as the two "Entotheonella" phylotypes from T. swinhoei from the Japan Sea. Genomic features related to utilization of various carbon sources, peptidase secretion, $\mathrm{CO}_{2}$ fixation, sulfate reduction, anaerobic respiration, and denitrification indicated the mixotrophic nature of "Entotheonella." The endospore-forming potential along with metal- and antibiotic resistance indicated "Entotheonella" was highly resilient to harsh conditions. The potential for endospore formation also explained the widespread distribution of "Entotheonella" to some extent. The discovery of Type II (general secretion pathway proteins and the Widespread Colonization Island) and Type VI secretion systems in "Entotheonella" suggested it could secrete extracellular hydrolases, form tight adhesion, act against phagocytes, and kill other prokaryotes. Overall, the newly discovered genomic features suggest "Entotheonella" is a highly competitive member of the symbiotic community of $T$. swinhoei.

Keywords: sponge symbiont, "Entotheonella,” metagenome binning, mixotroph, resilience, interaction

\section{INTRODUCTION}

The study of sponge microbiology has long been driven by the need for marine drug discovery (Piel et al., 2004; Wilson et al., 2014). Symbionts of marine sponges have become a fascinating field, due to their fidelity to hosts, unique phylogenetic patterns, importance in biogeochemical cycles, and great biotechnology potential (Taylor et al., 2007; Hentschel et al., 2012). To date, more than 50 phyla of microbial symbionts have been discovered from marine sponges based on pyrosequencing 
(Webster et al., 2010; Schmitt et al., 2012; Reveillaud et al., 2014). However, most sponge microbial symbionts remain uncultured, which limits our understanding of their metabolism and functions. Metagenome binning represents an approach that overcomes the difficulty of cultivating microbes and can reveal the unknown genomic features of uncultured microbes (Albertsen et al., 2013). Application of metagenome binning to sponge microbiology has yielded four draft genomes of "Candidatus Synechococcus spongiarum" (Gao et al., 2014; Burgsdorf et al., 2015), three draft genomes of autotrophic symbionts from deep-sea glass sponge (Tian et al., 2016), and two draft genomes of candidate genus "Entotheonella" (Wilson et al., 2014), which have greatly extended our knowledge of the functions of uncultured symbionts in sponges.

Theonella swinhoei and its Gram-negative, filamentous symbiont, "Entotheonella," represent the most prolific and wellstudied host-symbiont pair in terms of natural product isolation, chemical localization, symbiont identification, and PKS/NRPS gene cluster studies (Bewley et al., 1996; Bewley and Faulkner, 1998; Schmidt et al., 2000; Piel et al., 2004; Wilson et al., 2014; Ueoka et al., 2015; Freeman et al., 2016). A recent major advancement was the recovery of "Entotheonella" genomes from the metagenome of $T$. swinhoei from the Japan Sea, namely "Candidatus Entotheonella factor" TSY1 and "Candidatus Entotheonella gemina” TSY2 (Wilson et al., 2014). The genomic analysis provided confirmative evidence that almost all bioactive polyketides and non-ribosomal peptides derived from T. swinhoei are synthesized by "Entotheonella."

Nonetheless, compared with the comprehensive knowledge of the secondary metabolism repertoire of "Entotheonella," little is known about its other metabolic features. The large genome size ( $>9 \mathrm{Mb})$ and candidate taxonomic status make its genome annotation very difficult. Additionally, the wide distribution of "Entotheonella" in sponges raises more questions about its genetic diversity and ecological roles (Wilson et al., 2014). Clearly, more "Entotheonella" genomes are needed for a better understanding of this uncultured sponge symbiont.

In this study, two "Entotheonella" genomes were obtained from the metagenome of the sponge T. swinhoei from the South China Sea, which showed high coherence to the two other genomes derived from the Japan Sea (Wilson et al., 2014). Based on the annotations of the four genomes, the lifestyle of "Entotheonella," resilience, and interaction with the sponge host/other microbes are discussed. The results suggest that "Entotheonella" is a highly competitive member of the symbiotic community of T. swinhoei.

\section{MATERIALS AND METHODS}

\section{Sample Collection, DNA Extraction, and Metagenome Sequencing}

Individuals $(n=3)$ of $T$. swinhoei (yellow interior) were randomly sampled by scuba diving within a $15 \mathrm{~m}$ radius at approximately $10 \mathrm{~m}$ depth near Yongxing Island $\left(112^{\circ} 20^{\prime} \mathrm{E}\right.$, $16^{\circ} 50^{\prime} \mathrm{N}$ ) in the South China Sea, and were morphologically identified by Prof. Jinhe Li at the Institute of Oceanology, Chinese
Academy of Sciences. Sponge tissues were quickly rinsed with sterile artificial seawater then cut into small pieces thinner than $5 \mathrm{~mm}$. Subsequently, the specimens were fixed in RNA Later ${ }^{\circledR}$ (Qiagen, Hilden, Germany) at $4^{\circ} \mathrm{C}$ for $8 \mathrm{~h}$. The fixed specimens were then transported to the lab on ice. Total DNA was extracted with a QIAGEN DNeasy Tissue Mini kit (Qiagen), following the manufacturer's instructions. The integrity of DNA samples was inspected by $0.8 \%$ agarose gel electrophoresis. DNA samples of good quality (concentration $>50 \mathrm{ng} / \mu \mathrm{l}, 1.8<A_{260} / A_{280}<2.0$ ) were pooled and used for metagenome sequencing.

Metagenome sequencing was carried out at Genewiz, Inc (Beijing, China). In brief, a library with a 350 bp insert length was constructed and sequenced on a HiSeq 2000 (Illumina, USA), following the manufacturer's instructions. Metagenome reads in FASTQ format (approximately $40 \mathrm{~Gb}$ ) were trimmed using a minimum Phred score of 20 and a minimum length of 36, by Trimmomatic 0.32 (Bolger et al., 2014), allowing no ambiguous nucleotides or adaptors. Finally, about $28 \mathrm{~Gb}$ reads $(125,866,412$ paired-end reads and 47,841,730 single-end reads) were fed into metaVelvet for assembly (kmer $=57$, minimum contig length $=300 \mathrm{bp}$; Namiki et al., 2012). The metagenome assembly used for metagenome binning included 371,342 contigs with a minimum length of $500 \mathrm{bp}$.

\section{Metagenome Binning, Taxonomic Assignment, and Genome Annotation}

MetaBAT, an automatic metagenome binning software package, was used for metagenome binning (Kang et al., 2015). Metagenomic contigs no shorter than $2 \mathrm{~kb}$ were binned based on empirical probabilistic genome abundance and tetranucleotide frequency. According to the recommended protocol, the sensitive mode was applied first to recruit contigs then the specific mode was applied to strip off non-targeting contigs. As rRNA operons were often lost in the binning process due to their high coverage, a whole-genome tetra-correlation-search-based platform, JSpeciesWS Online, was used to find the targeting genomes (Richter et al., 2016). Average nucleotide index (ANI) was calculated to determine whether two genomes were closely related, using the script ani.rb with default settings ${ }^{1}$. The completeness of binned genomes was estimated based on the presence/absence of 106 essential bacterial genes, using the script HMM.essential.rb ${ }^{1}$.

The metagenome bins and publicly available "Entotheonella" genomes (AZHW01000000 and AZHX01000000) were annotated using a RASTtk pipeline based on the SEED subsystem (Brettin et al., 2015). Primary coding sequence (CDS) prediction and annotation was done by the scripts rast-callfeatures-CDS-glimmer3, rast-call-features-CDS-prodigal, and rast-annotate-proteins-kmer-v2, followed by rast-annotateproteins-kmer-v1 - $\mathrm{H}$ to further annotate hypothetical proteins. Additionally, rast-call-features-ProtoCDS-kmer-v2 and rastcall-features-ProtoCDS-kmer-v1 were applied, as gene calling might have been missed in some regions. The PATRIC web service was used as the primary comparative analysis platform, in which KEGG pathways were implemented (Wattam et al.,

\footnotetext{
${ }^{1}$ https://github.com/lmrodriguezr/enveomics-gui
} 
2014). Bidirectional BLAST analysis was used to define the core gene set and singletons (genes without any hit against any other genome than their own) of four "Entotheonella" genomes based on the EDGAR platform (Blom et al., 2009). In bidirectional BLAST analysis, "Candidatus Entotheonella factor" TSY1 was used as reference owing to its high completeness. Scripts used for metagenome binning and genome annotation are provided in the Supplementary Information.

\section{Phylogenetic Analysis of Phosphoenolpyruvate Carboxylase (PEPC)}

The BLASTP program was used to determine the sequences that were most closely related to "Entotheonella" phosphoenolpyruvate carboxylase (PEPC) sequences, with an $e$-value cutoff of $10^{-5}$, against the non-redundant protein database of NCBI (Altschul et al., 1990). For phylogenetic analysis, a total of 277 reviewed PEPC sequences were retrieved from the Uniprot database (Consortium, 2015). A second round of BLASTP analysis was carried out using downloaded PEPC sequences as the local database to discard sequences that were largely distant to "Entotheonella," resulting in a reference dataset of 55 high-quality sequences ranging from bacteria to higher plants. Sequences were then aligned using MUSCLE (Edgar, 2004). The alignment was then manually inspected and corrected, resulting in a final alignment with 364 amino acid sites included. A maximum-likelihood tree was reconstructed based on the JTT $+\mathrm{G}$ model with a bootstrap value of 1000 in MEGA6 (Tamura et al., 2013).

\section{Nucleotide Sequence Accession Numbers}

The draft genomes of $\mathrm{v} 4.2$ and $\mathrm{v} 4.3$ were deposited at the European Nucleotide Archive (ENA) under accession PRJEB12598 and PRJEB12599, respectively. Additionally, the annotated genomes are available from the RAST guest account ${ }^{2}$ (username guest; password guest) with accession numbers 93171.12 for v4.2 and 93171.13 for v4.3.

\section{RESULTS AND DISCUSSION}

\section{Genome Recovery}

Metagenome bins generated by MetaBAT with a minimum size of $0.5 \mathrm{Mb}$ were submitted to JSpeciesWS Online to target taxa of interest. Two metagenome bins, v4.2 and v4.3, were found to be closely related to the two known "Entotheonella" phylotypes/species, respectively (Wilson et al., 2014). Based on the correlation Z-score to all published whole and draft genomes, bin v4.2 and "Candidatus Entotheonella gemina" TSY2 (hereafter TSY2) had a Z-Score of 0.995 , while the Z-score between bin v4.3 and "Candidatus Entotheonella factor" TSY1 (hereafter TSY1) reached 0.996. A Z-score $>0.989$ suggested two strains were closely related. The ANI between v4.2 and TSY2 was $99.90 \%$.

${ }^{2}$ http://rast.nmpdr.org/
TABLE 1 | General information for metagenome bins obtained in this study and their close relatives.

\begin{tabular}{|c|c|c|c|c|}
\hline Taxon $^{a}$ & v4.3 & TSY1 & v4.2 & TSY2 \\
\hline Genome size (Mb) & 6.1 & 8.9 & 7.9 & 8.5 \\
\hline $\mathrm{G}+\mathrm{C}$ content $(\%)$ & 56.48 & 55.79 & 56.26 & 55.55 \\
\hline N50 (kb) & 5.1 & 8.4 & 6.9 & 4.2 \\
\hline No. of contigs & 1354 & 1596 & 1378 & 2592 \\
\hline No. of CDSs ${ }^{b}$ & 6618 & 8397 (8438) & 8752 & 8748 (8989) \\
\hline Completeness (\%) & 73.6 & 93.4 & 82.1 & 77.3 \\
\hline No. of hypothetical proteins ${ }^{b}$ & 4063 & 4790 (7096) & 5772 & $5359(7935)$ \\
\hline No. of seed subsystems & 766 & 828 & 813 & 839 \\
\hline
\end{tabular}

$a_{\text {V4.3, v4.2 and were obtained in this study. TSY1 (AZHW01000000) and TSY2 }}$ (AZHX01000000) were from the Japan Sea sponge Theonella swinhoei. ' ${ }^{\text {Genomes }}$ of TSY1 and TSY2 were re-annotated in this study using a RASTtk pipeline. The counts of CDSs and hypothetical proteins derived from a RefSeq pipeline are shown in parentheses.

Meanwhile, the ANI between v4.3 and TSY1 was 99.91\%. ANI higher than $96 \%$ strongly indicates two genomes represent the same species (Richter and Rosselló-Móra, 2009).

General information for binned genomes and their close relatives is summarized in Table $\mathbf{1}$. Compared with TSY1, the completeness of $\mathrm{v} 4.3$ was lower, whereas $\mathrm{v} 4.2$ had greater completeness than TSY2. The contamination (multiple copies of essential genes) rates of $\mathrm{v} 4.2$ and $\mathrm{v} 4.3$ were both less than $4 \%$. Overall, the quality of the two metagenome bins in this study met the requirement for reliable genome annotation and comparison with TSY1 and TSY2. Due to the high genome sequence similarities between genomes from the Japan Sea and the South China Sea, the functional annotation profiles of the genomes from the two geographical locations were reasonably coherent. Also, because of the considerable number of hypothetical proteins, the annotation profiles of the two "Entotheonella" phylotypes were largely similar. Hence, the subsequent analysis focused on the shared features of all four genomes, which comprised $62.4 \%$ of the total unique features (3182 out of 5099 non-hypothetical proteins). In this study, we re-annotated TSY1 and TSY2, which were previously annotated by a RefSeq pipeline (Wilson et al., 2014). In contrast to the RefSeq pipeline, the RASTtk pipeline consisted of steps of re-annotating hypothetical proteins and an algorithm for alternate gene calling. Therefore, the proportion of hypothetical proteins derived from the RASTtk pipeline was less than that from the RefSeq pipeline. The functions that could not be annotated by the RefSeq pipeline but were annotated by the RASTtk pipeline are listed in Supplementary Data Sheet 1 .

According to the output of EDGAR, the pan genome of "Entotheonella" contained 18,825 genes, 13,942 of which were annotated as hypothetical proteins. The core gene set contained 2946 genes, 1672 of which were annotated as hypothetical proteins (Supplementary Data Sheet 2). Besides the core gene set, we identified 1648, 1889, 678, and 459 singletons from TSY1, TSY2, v4.2, and v4.3, respectively. However, only 447 (9.6\%) of the singletons were not hypothetical proteins. Annotations 
of these 447 genes are provided in Supplementary Data Sheet 3.

\section{Mixotrophy of "Entotheonella"}

In general, "Entotheonella" contains the genomic capacity for an aerobic heterotrophic lifestyle. Near-complete pathways for glycolysis, the tricarboxylic acid cycle (TCA), the pentose phosphate pathway, and oxidative phosphorylation could be identified from the four "Entotheonella" genomes, including v4.2 and v4.3 from the South China Sea, and TSY1 and TSY2 from the Japan Sea (Supplementary Figure S1). The SEED subsystem used the general term "feature" to describe any genomic region with some annotated functions. According to the feature counts, about $10 \%$ of "Entotheonella" features were related to the utilization of various carbon sources. For carbon source utilization, the most abundant subsystem was "Chitin and N-acetylglucosamine utilization" (Figure 1). The highly efficient uptake of dissolved and particulate organic matter by sponges could provide the microbial communities with various forms of carbon source (De Goeij et al., 2013). The ability to utilize a wide range of carbon sources could be beneficial for "Entotheonella." The capacity to degrade recalcitrant carbon sources (e.g., chitin and cellulose) would also benefit the host and other symbiotic members by increasing the availability of more labile carbon sources. Similarly, the ability to degrade $\mathrm{N}$-acetylglucosamine was found in another iconic phylum of sponge symbionts, "Poribacteria" (Siegl et al., 2011). The source of chitin and cellulose in the sponge mesohyl may be food particles, which are derived from particulate organic matter taken up by pinacocytes and choanocytes of sponges (Maldonado et al., 2012).

Urea and other nitrogen-containing metabolic products are considered to be important nitrogen sources of sponge symbionts (Webster and Thomas, 2016). Urease (EC 3.5.1.5), urease accessory proteins, urea channels, and urea transporters were found in all "Entotheonella" genomes. We also investigated the proteinogenic amino acid biosynthesis potential; however, we only found features related to the biosynthesis of Cys, Met, Lys, Thr and Ser, implying "Entotheonella" relies on exogenous amino acid supply, which could be available from its sponge host or the symbiotic microbiota. Accordingly, we found 11 peptidases that were present in all "Entotheonella" genomes. These peptidases belonged to aminopeptidases (EC 3.4.11.-), metallocarboxypeptidases (EC 3.4.17.-), omega peptidases (EC 3.4.19.-), serine endopeptidase (EC 3.4.21.-), and signal peptidase. Features related to peptide transportation, such as $\mathrm{ABC}$ transporters of oligopeptides (TC 3.A.1.5.1), dipeptides (TC 3.A.1.5.2), and branched-chain amino acids (TC 3.A.1.4.1) were also found in all "Entotheonella" genomes.

Besides the heterotrophic traits, the existence of RuBisCO indicated the carbon fixation potential of "Entotheonella." A nearcomplete Calvin-Benson cycle was reconstructed in this study (Figure 2). Some missing genes could be due to incompleteness of the current genomes. Carbonic anhydrase (EC 4.2.1.1) was also found in all "Entotheonella" genomes. This enzyme aids in concentrating $\mathrm{CO}_{2}$ by catalyzing $\mathrm{CO}_{2}$ hydration, a simple but

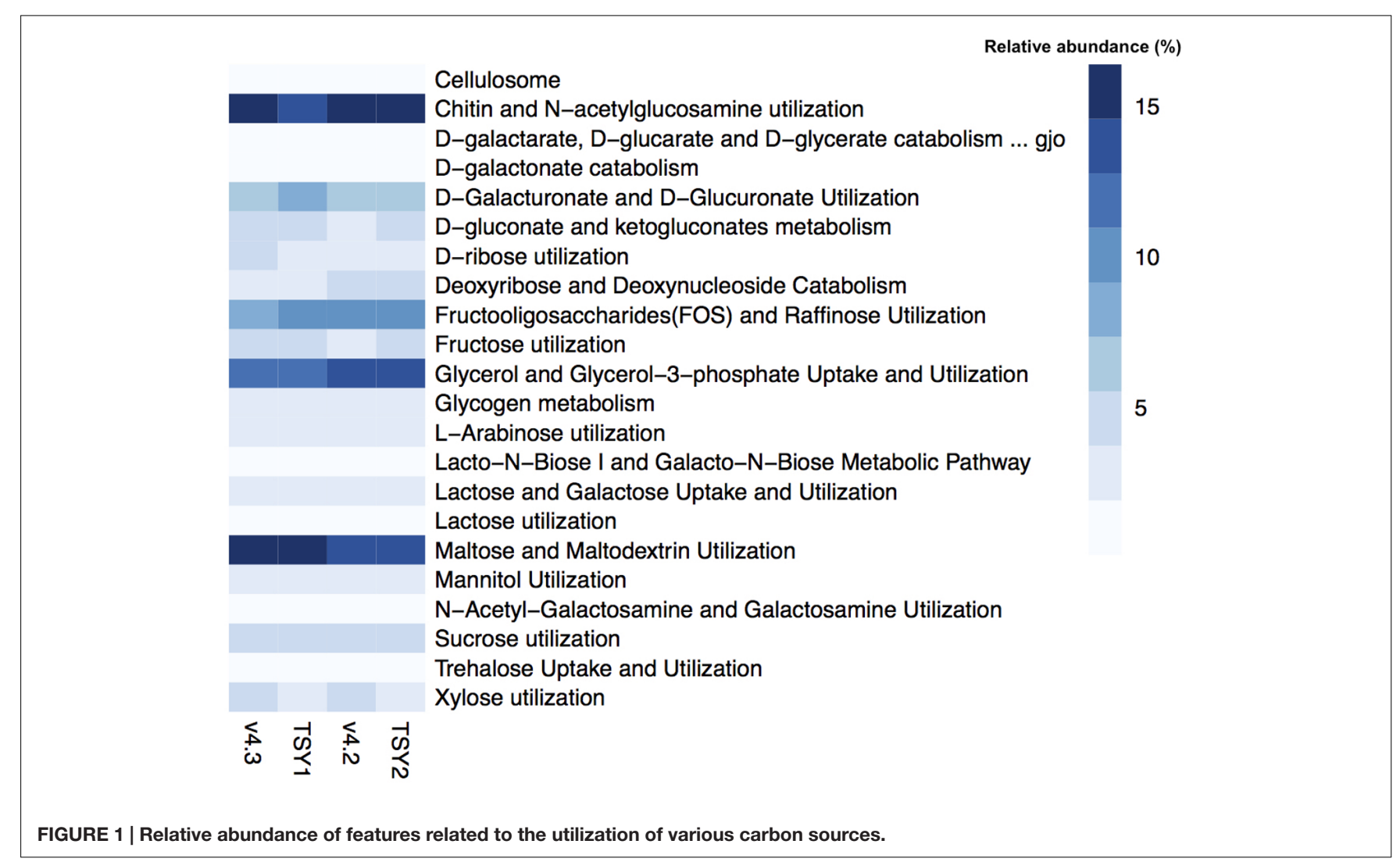




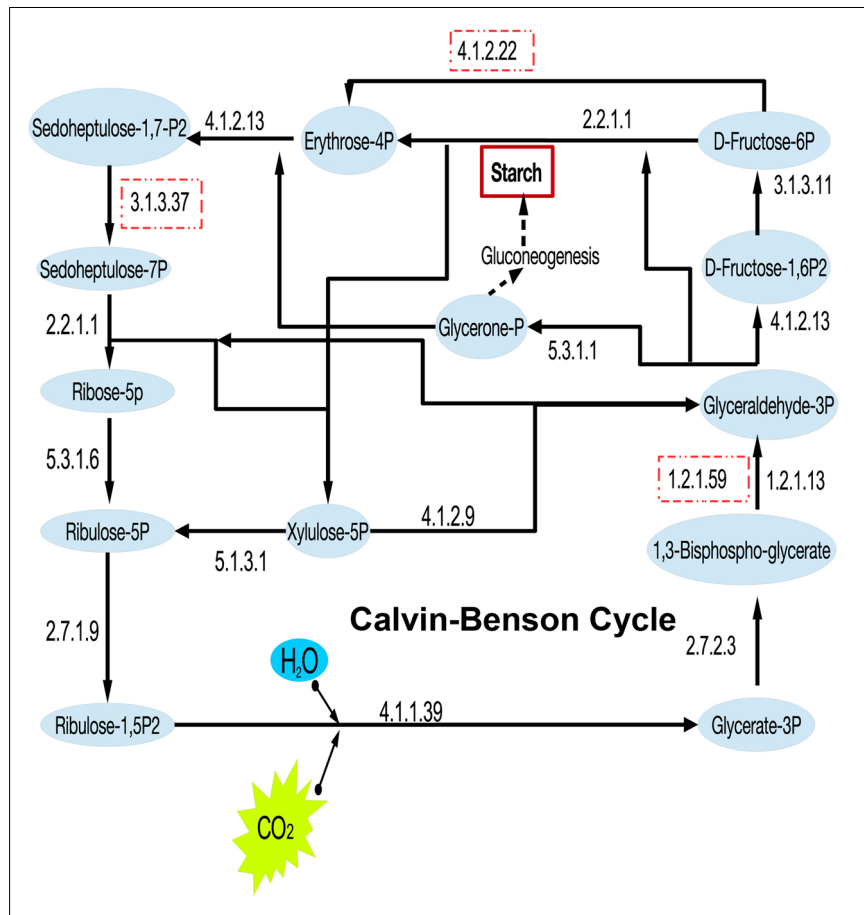

FIGURE 2 | Reconstruction of the Calvin-Benson cycle in "Entotheonella". The EC numbers of key enzymes are shown. The dashed squares indicate the enzyme was missing in at least one genome.

physiologically relevant reaction in all kingdoms of life (Capasso and Supuran, 2015).

Interestingly, a set of genes related to crassulacean acid metabolism (CAM) and the $\mathrm{C}_{4}$-dicarboxylic acid cycle existed in all "Entotheonella" genomes. It is known that CAM and the $\mathrm{C}_{4}$-dicarboxylic acid cycle greatly enhance $\mathrm{CO}_{2}$ concentration in higher plants. Nonetheless, particular tissue structures and complex regulation mechanisms are involved in CAM and the $\mathrm{C}_{4}$-dicarboxylic acid cycle of plants (Dodd et al., 2002; Langdale, 2011). For "Entotheonella," a possible scenario is that these enzymes coexist for unknown reasons and function in other metabolic pathways, as none of the enzymes is unique to plants. By searching KEGG reference genomes, we found that genes related to CAM existed in the genome of the legume symbiont Methylobacterium nodulans as well (Jourand et al., 2004). The key enzyme of CAM and the $\mathrm{C}_{4}$-dicarboxylic acid cycle is PEPC (Christin et al., 2014). BLAST analysis showed the PEPC sequences of "Entotheonella" were most similar to those of the halophilic Gammaproteobacteria (Arhodomonas and "Spiribacter"; Adkins et al., 1993; López-Pérez et al., 2013). Phylogenetic analysis showed PEPC of "Entotheonella" differed from the homologs of "Spiribacter" and Arhodomonas, although they shared high sequence similarity (Figure 3). The placement of "Entotheonella" PEPC was divergent from the Gammaproteobacteria and Viridiplantae, implying the unique evolutionary traits of "Entotheonella."

With a predicted genome size over $9 \mathrm{Mb}$, "Entotheonella" belongs to the bacteria with large genomes (Wilson et al., 2014; and the data $\left.{ }^{3}\right)$. The mechanisms that support the large genomes of "Entotheonella" are worth mining. DNA replication is typically demanding with respect to nutrients and energy. For microbes inhabiting niches with scarce nutrients, genome streamlining reduces the minimum requirement for reproduction and thus promotes survival (Giovannoni et al., 2014). Experiments have proven marine sponges can effectively uptake dissolved and particulate organic matter in seawater (Yahel et al., 2003; De Goeij et al., 2008, 2013; Hadas et al., 2009). The organic matter might subsequently serve as communal food particles for sponge cells and microbes. We speculated that "Entotheonella" could benefit from communal food particles. Meanwhile, the potential for $\mathrm{CO}_{2}$ fixation offers an alternative strategy when carbon sources are in short supply, e.g., competitive carbon uptake from other symbionts. More experiments are needed to determine how important $\mathrm{CO}_{2}$ fixation is to "Entotheonella" and the whole symbiotic community of T. swinhoei.

Low DNA G+C content $(<30 \%)$ is considered an adaptive trait of planktonic bacteria when facing limited nitrogen sources (Luo and Moran, 2015). With G+C content over 55\%, the replication of "Entotheonella" DNA is not only energy demanding but also nitrogen demanding. Marine sponges have been shown to serve as a net nitrogen source through remineralization of particulate organic matter (Maldonado et al., 2012). Hence, "Entotheonella" in sponge mesohyl may face less nitrogen limitation than those low $\mathrm{G}+\mathrm{C}$ content planktonic bacteria. In addition to the inorganic nitrogen sources, the symbiotic community may also provide organic nitrogen sources for "Entotheonella." The organic nitrogen sources in sponge mesohyl could be the leaky products from the metabolism of other symbionts (Giovannoni et al., 2014) and the sponge cell detritus (De Goeij et al., 2013).

\section{Adaption of "Entotheonella" to Anaerobic Conditions in the Sponge Body}

Apart from carbon fixation potential, "Entotheonella" genomes encode enzymes for sulfate reduction. A near complete dissimilatory sulfate reduction pathway was reconstructed from all "Entotheonella" genomes (Figure 4). The dissimilatory sulfate reduction potential may supply the energy that is needed in the Calvin-Benson cycle. Compared with the newly found sulfur-oxidizing bacterium in deep-sea glass sponge (Tian et al., 2016), TSY1 and v4.3 lacked dissimilatory sulfite reductase (EC 1.8.99.5), which might be due to the incompleteness of the genomes. In addition, the Sox complex and a complete assimilatory sulfate reduction pathway were found in all four genomes. When "Entotheonella" was recognized as a genus of Deltaproteobacteria, a mixed culture of "Entotheonella" and other bacteria could be acquired using media designed for sulfate-reducing bacteria (Schmidt et al., 2000). Our finding of sulfate-reduction potential in "Entotheonella" echoed this research. Anaerobic zones existed in actively pumping sponges, and activities of both sulfate reduction and

\footnotetext{
${ }^{3}$ http://www.genomesize.com/prokaryotes
} 


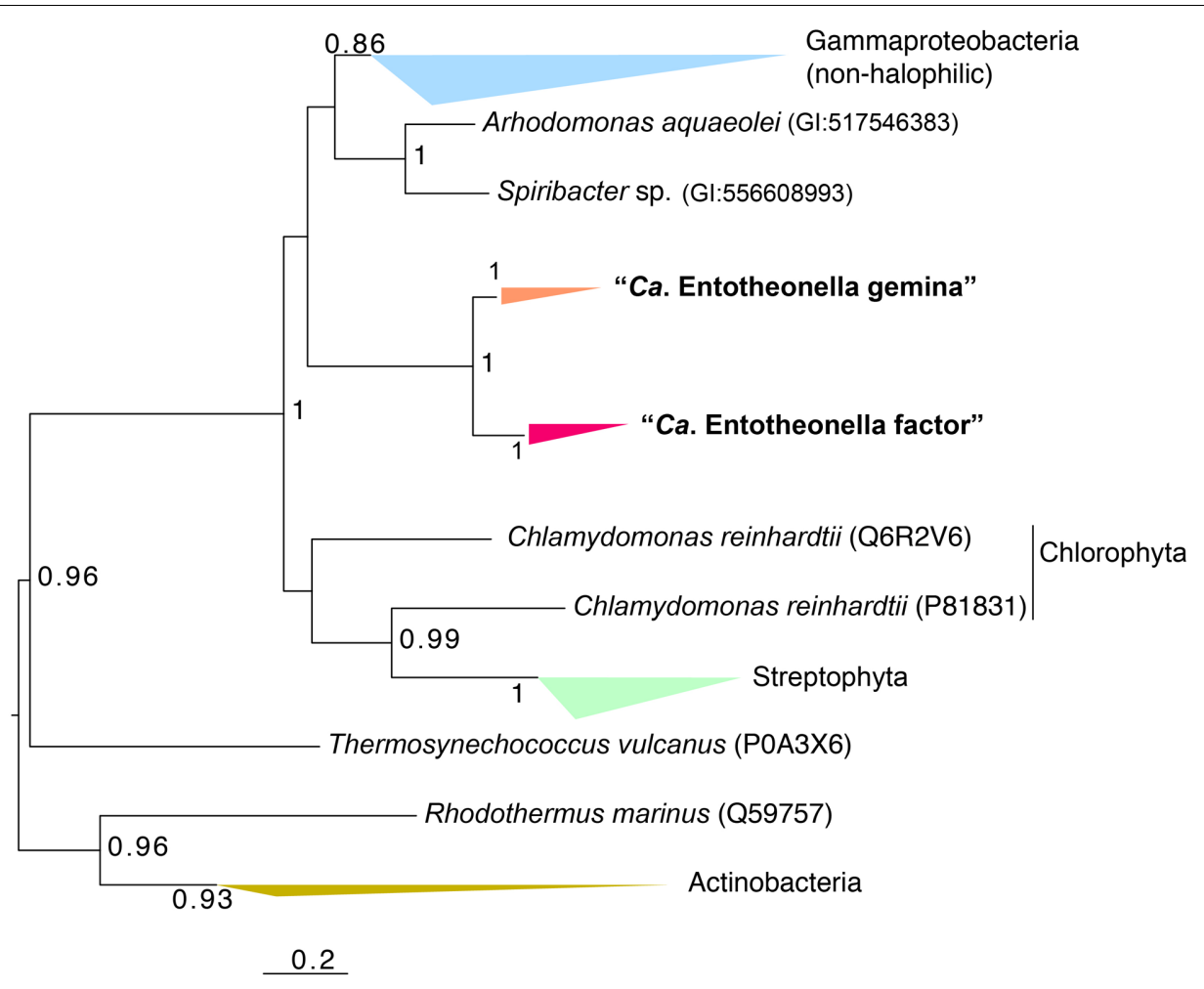

FIGURE 3 | Maximum-likelihood phylogenetic tree of phosphoenolpyruvate carboxylase (PEPC) genes of "Entotheonella". Bootstrap values over 0.5 are shown. For sequences retrieved from the Uniprot database, the Uniprot ID is shown in parentheses.

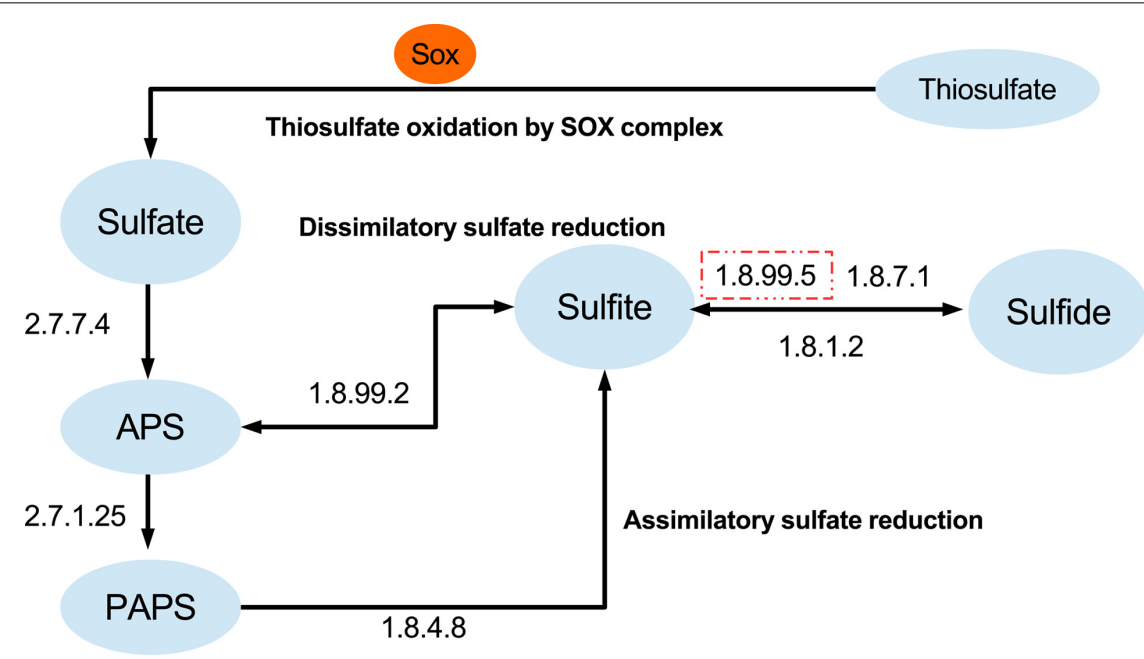

FIGURE 4 | Sulfate-reducing and sulfur-oxidizing metabolism capacity of "Entotheonella". The dashed square indicates the enzyme was missing in at least one genome.

anaerobic ammonia oxidation were detected (Hoffmann et al., 2005; Mohamed et al., 2010). The chemotrophic ability of "Entotheonella" ensures its energy supply when oxygen is scarce. Additionally, carboxylic acids derived from the sulfate reduction process might be transferred to the host (Hoffmann et al., 2005).
Features related to anaerobic respiratory reductases were detected in "Entotheonella." The features shared by all "Entotheonella" genomes included flavodoxin reductases, anaerobic dehydrogenases, and ferredoxin reductase. Interestingly, "Entotheonella" possessed Nar, Nir, Nor, and Nos gene clusters, which implied it could complete anaerobic respiration 


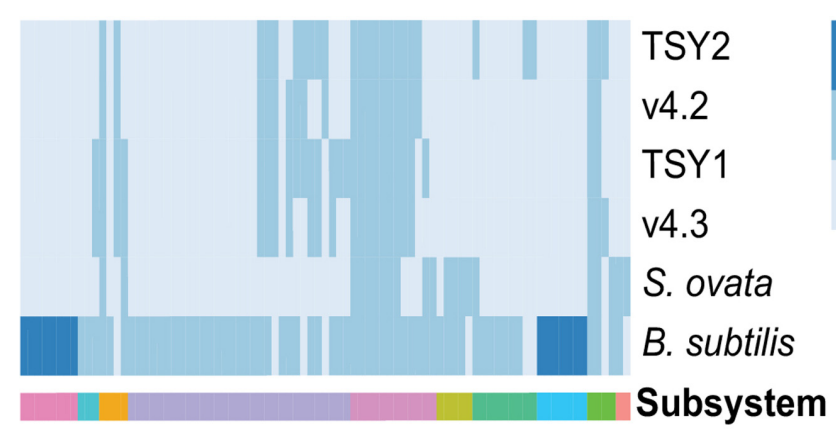

Bacillus sporulation killing factor A biosynthetic cluster
Small acid-soluble spore proteins
Spore core dehydration
Spore germination
Sporulation cluster
Sporulation draft
Sporulation gene orphans
Sporulation-associated proteins with broader functions
SpoVS protein family
Stage III sporulation protein A

FIGURE 5 | Comparison of sporulation features across B. subtilis (GCA_000009045.1), Sporomusa ovata (ASXP01000000), and four "Entotheonella" genomes. $B$. subtilis and Sporomusa ovata were re-annotated using a RASTtk pipeline.

through denitrification. Additionally, NnrA was found only in "Candidatus Entotheonella factor," i.e., TSY1 and v4.3.

Adaptive traits of sponge symbionts to anaerobic conditions are not confined to "Entotheonella." A recent study showed that a combination of aerobic and microaerophilic states could increase the diversity and novelty of cultivable microbes from T. swinhoei, which suggested that the microbes in T. swinhoei faced variable oxygen levels (Lavy et al., 2014). Study of six sponge metagenomes has highlighted the enrichment of adaptive traits to anaerobic conditions, such as nitrate respiration (Fan et al., 2012). In "Poribacteria," genomic traits related to a lifestyle under anaerobic conditions, including nitrite reductase (EC 1.7.2.1), nitric oxide reductase (EC 1.7.99.1), and the Wood-Ljungdahl pathway (anoxic $\mathrm{CO}_{2}$ fixation) have been found (Siegl et al., 2011).

\section{Resilience of "Entotheonella"}

Features of endospore formation were found in "Entotheonella" genomes. Until now, the only known Gram-negative bacterium that could form endospores was Sporomusa ovata (Poehlein et al., 2013). In comparison with $S$. ovata and the model species Bacillus subtilis, "Entotheonella" genomes possessed the coding potential for spore core dehydration, spore germination, and sporulation clusters (Figure 5). Bacterial endospores are the most durable cells in nature (Nicholson et al., 2000). The potential for endospore formation could strengthen the resistance of "Entotheonella" against environmental stress and increase its ability to thrive in a diverse range of niches. One possible scenario is that $T$. swinhoei harbors dense microbiota, and hence the intra-/inter-species competition is fiercer than that in planktonic communities. By forming endospores, some "Entotheonella" cells could survive and relocate to other hosts.

The "Entotheonella" 16S rRNA gene could be detected in sponges and seawater from different geographical locations (Wilson et al., 2014). However, microscopic evidence for the presence of "Entotheonella" has been found only in lithistid sponges (e.g., Theonella and Discodermia; Schmidt et al., 2000; Brück et al., 2008; Wilson et al., 2014). For instance, Aplysina aerophoba yielded PCR products of the "Entotheonella" 16S rRNA gene but no filamentous bacteria were observed during transmission electron microscopy (Friedrich et al., 2001). Based on the sporulation-related features of "Entotheonella," we hypothesized that its spores are widespread and the unknown cue for spore germination lies in Lithistida sponges. The genetic elements encoding the endospore-forming process are flexible in composition, conserved in evolution, and sophisticated in regulation. Even in the well-studied B. subtilis, more information is needed to fully understand the endospore-forming process (Hutchison et al., 2014). Sporulation-related features in the "Entotheonella" genomes may only represent the tip of its development course. It would be interesting to monitor the development of T. swinhoei and investigate when its filamentous partner appears.

"Entotheonella" possessed abundant genomic features of metal resistance, including resistance to $\mathrm{As}, \mathrm{Co}, \mathrm{Cu}, \mathrm{Hg}$, and $\mathrm{Zn}$. Beta-lactamase and multi-drug resistance efflux pumps were the main mechanisms of antibiotic resistance. A recent attempt at culturing T. swinhoei symbiotic bacteria yielded 12 operational taxonomic units that were tolerant to high arsenic 
A

T2SS

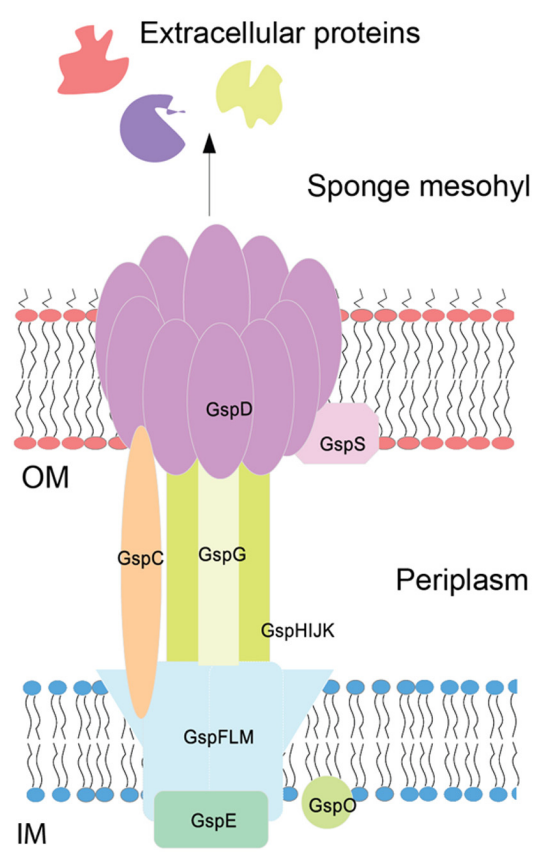

"Entotheonella" cytoplasm
B

T6SS
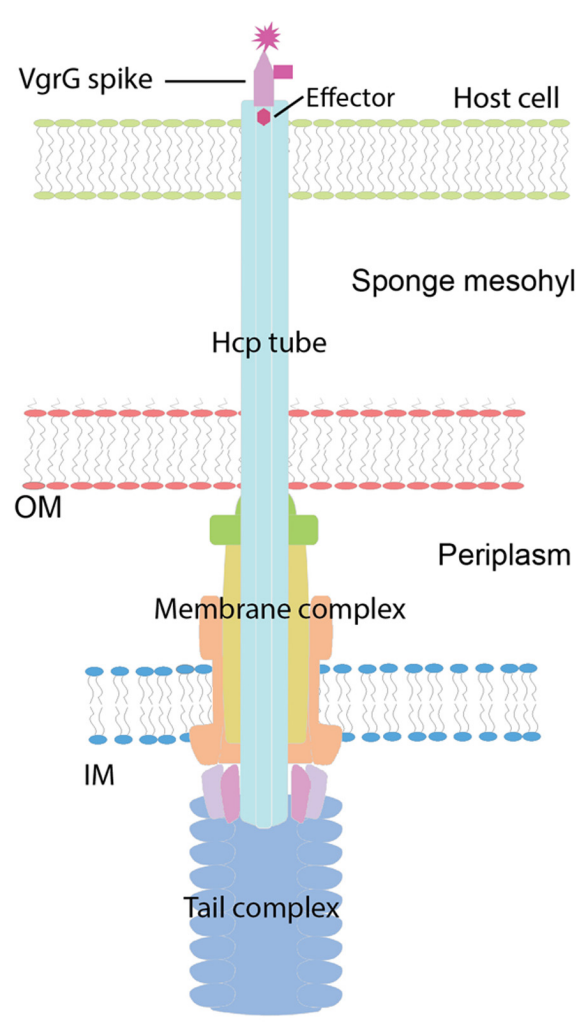

"Entotheonella" cytoplasm

FIGURE 6 | Schematic view of the structures and mechanisms of Type II (T2SS) (A) and Type VI secretion systems (T6SS) (B). The structural assembly was predicted based on the gene content of "Entotheonella" draft genomes and visualized based on the KEGG diagram and the review of Costa et al. (2015). OM, outer membrane; IM, inner membrane. According to the review of Costa et al. (2015), in T2SSs, the GspC protein recruits folded exoproteins from the periplasm to secretin (GspD). GspE acts as ATPase and facilitates the assembly of the periplasmic pseudopilus (GspG) through the IM. The exoproteins are pushed by the pseudopilus through the secretin channel. In T6SSs, effectors are recruited by VgrG and pass through the haemolysin co-regulated protein (Hcp) tube. The sheath contraction is triggered by an unknown extracellular signal, resulting in the ejection of the effectors into targeting cells.

concentrations (Keren et al., 2015). The resistance to As and other heavy metals found within 'Entotheonella' might be beneficial not only for these organisms but also for the $T$. swinhoei symbiotic community, as sponges and their symbionts are frequently exposed to toxic matter in seawater (De Goeij et al., 2013).

\section{Interaction between "Entotheonella" and Other Organisms}

Eukaryotic-like proteins (ELPs) are well known as important factors involved in sponge-microbe interactions (Fan et al., 2012; Nguyen et al., 2014). In "Entotheonella" genomes, ELPs such as ankyrin repeat proteins (ARP), leucine-rich repeats (LRR), and tetratricopeptide repeats (TPR) were found (Supplementary Table S1). Other than ELPs, secretion systems of Gram-negative bacteria play key roles in response to environmental factors and interaction with other macro-/micro-organisms (Costa et al., 2015). Type II secretion systems (T2SSs) and Type VI secretion systems (T6SSs) represented the major protein secretion systems unveiled in this study (Supplementary Table S2; Figure 6).

In terms of T2SSs, we found general secretion pathway proteins (Gsps) and features related to the Widespread Colonization Island. T2SSs are found in both clinical pathogens and non-pathogens and usually require $12-15$ Gsps to form the double-membrane-spanning structure (Costa et al., 2015). In our study, we found 14 Gsps from v4.2, v4.3, and TSY1. For unknown reasons, TSY2 was devoid of Gsps. T2SSs secrete folded proteins from the periplasm into the extracellular environment (Figure 6A). Extracellular proteins/enzymes are important for microbes to utilize the organic matter in a certain niche or interact with other organisms. Some extracellular proteolytic enzymes, mainly of the metalloprotease group, have been found to be virulence factors (Costa et al., 2015). Only one secreted collagenase (EC 3.4.24.3) was found 
in "Entotheonella," whereas the known sponge pathogen Pseudoalteromonas agarivorans NW4327 contained 30 secreted serine proteases and metalloproteases (Choudhury et al., 2015). Here we hypothesize that the ability to degrade collagen might be essential for "Entotheonella" to obtain space in the sponge mesohyl.

The Widespread Colonization Island represents an ancient and a new subtype of Type II secretion, which encompasses the tad (tight adherence) locus for colonization of surfaces and biofilm formation (Planet et al., 2003). The Widespread Colonization Island also represents a hotspot of horizontal gene transfer, which might function in the evolution and diversification of "Entotheonella." In a study of Actinobacillus, at least 12 of the tad genes were necessary to generate the adherence-related phenotypes (Tomich et al., 2007). In this study, we identified features related to 17 tad genes, suggesting "Entotheonella" could form tight adherence, which might contribute to its stable residence in sponges or other types of host-microbe interactions. Nevertheless, the "Entotheonella" cells were free of fibrils and flagella (Wilson et al., 2014). The regulation and structure of the "Entotheonella"-derived tad locus need further investigation.

T6SSs were first discovered in Vibrio cholerae (Pukatzki et al., 2006). The needle-like structure could inject effectors and toxic proteins into other cells (mostly host/eukaryotic cells) and then kill the cells (Figure 6B). All the core components of T6SSs (Boyer et al., 2009) were detected in "Candidatus Entotheonella gemina" (TSY2 and v4.2), whereas the spike protein VgrG was missing in "Candidatus Entotheonella factor" (TSY1 and v4.3). A previous study of the sponge pathogen P. agarivorans NW4327 revealed the existence of 19 genes related to T6SSs (Choudhury et al., 2015). This study is the second report of T6SSs in a sponge symbiont. Nevertheless, T6SSs are widespread in nature and not confined to known pathogens (Costa et al., 2015). In some cases, T6SSs can kill prokaryotic cells as well (Miyata et al., 2013). A recent pyrosequencing study of the protistan community in T. swinhoei revealed the existence of radiolarians, which might prey on symbiotic prokaryotes (He et al., 2014). We hypothesized that a potential benefit for "Entotheonella" of having T6SSs might be the ability to fight against zooplankton predators. Currently, there is no report of T. swinhoei disease and no "Entotheonella" features have been found under SEED subcategory "Toxins and superantigens." Its uncultured status largely restricts the biochemical and physiological study of "Entotheonella." How virulent "Entotheonella" could be to its hosts and how T6SSs are regulated in "Entotheonella" remain unknown. The present evidence does not support that T6SSs are related to the virulence of "Entotheonella." Nonetheless, it is likely that T6SSs make "Entotheonella" a competitive member of the symbiotic community.

\section{SUMMARY}

The uncultured status of "Entotheonella" means it remains cryptic in terms of life history, physiology, ecological roles, and association with its sponge host. The four draft genomes, despite being fragmentary and not thoroughly annotated, offered new insights into "Entotheonella" in T. swinhoei, e.g., mixotrophic traits (utilization of various carbon sources and $\mathrm{CO}_{2}$ fixation), adaptation to the anaerobic environment, resistance to threats, and interaction with other organisms (Table 2).

Despite the differences between the South China Sea and the Japan Sea in terms of temperature, salinity, and primary productivity, the "Entotheonella" strains from the two sea areas showed high intraspecies genomic coherence. Furthermore, future studies addressing the relationship between "Entotheonella" genetic diversity and host morphological features are essential for understanding the diversification of "Entotheonella." The interior of T. swinhoei investigated by Wilson et al. (2014) and us was yellow. T. swinhoei with a white interior was reported to harbor a different phylotype, "Candidatus Entotheonella serta" (genome not available; Ueoka et al., 2015). Comparative studies on white and yellow T. swinhoei genomes and their "Entotheonella" genomes will yield more valuable insights into symbiont diversification and sponge-symbiont interactions.

The genome information also provided clues for improving the strategy of cultivating "Entotheonella" in the lab. Firstly, genome analysis of "Entotheonella" suggests it does not favor

TABLE 2 | Summary of functional traits of "Entotheonella".

Functional traits

Calvin-Benson cycle

Utilization of various carbon sources

Anaerobic respiration

Denitrification

Sulfate reduction

Peptidase

Endospore formation

Metal resistance

Antibiotic resistance

Eukaryotic-like proteins

Type II secretion system

Widespread Colonization Island

Type VI secretion system
Interpretation

$\mathrm{CO}_{2}$ fixation

Adaptation to the diverse organic matter in sponge mesohyl

Energy supply in anoxic environment

Energy supply in anoxic environment

Energy supply in anoxic environment

Compensation for the reduced amino acid synthesis capacity

Resistance to environmental threats; widespread distribution

Resistance to environmental threats

Competition in symbiotic communities; resistance to environmental threats

Host-microbe recognition

Interaction with host environment

Tight adhesion

Host-microbe interaction, microbe-microbe competition 
one specific carbon source but can utilize a wide range of carbohydrates. The combination of various carbon sources might be important for its metabolism and growth. Secondly, although "Entotheonella" has a large and distinct secondary metabolism repertoire, it is able to synthesize only five proteinogenic amino acids (Cys, Met, Lys, Thr and Ser). Thus, providing free amino acids would be crucial for cultivating "Entotheonella" due to its deficiency in synthesizing all proteinogenic amino acids. Thirdly, the anaerobic respiration reductases and sulfatereduction capacity suggested "Entotheonella" faced variable oxygen levels in T. swinhoei. The shift between anaerobic and aerobic conditions might be important for the activity and metabolic regulation of "Entotheonella."

\section{AUTHOR CONTRIBUTIONS}

FL and ZL designed the study. FL undertook metagenome binning and genome analysis. JL finished the metagenome

\section{REFERENCES}

Adkins, J. P., Madigan, M. T., Mandelco, L., Woese, C. R., and Tanner, R. S. (1993). Arhodomonas aquaeolei gen. nov., sp. nov., an aerobic, halophilic bacterium isolated from a subterranean brine. Int. J. Syst. Bacteriol. 43, 514-520. doi: 10.1099/00207713-43-3-514

Albertsen, M., Hugenholtz, P., Skarshewski, A., Nielsen, K. L., Tyson, G. W., and Nielsen, P. H. (2013). Genome sequences of rare, uncultured bacteria obtained by differential coverage binning of multiple metagenomes. Nat. Biotechnol. 31, 533-538. doi: 10.1038/nbt.2579

Altschul, S. F., Gish, W., Miller, W., Myers, E. W., and Lipman, D. J. (1990). Basic local alignment search tool. J. Mol. Biol. 215, 403-410. doi: 10.1006/jmbi.1990.9999

Bewley, C. A., and Faulkner, D. J. (1998). Lithistid sponges: star performers or hosts to the stars. Angew. Chem. 37, 2162-2178. doi: 10.1002/(SICI)15213773(19980904)37:16<2162::AID-ANIE2162> 3.3.CO;2-U

Bewley, C. A., Holland, N. D., and Faulkner, D. J. (1996). Two classes of metabolites from Theonella swinhoei are localized in distinct populations of bacterial symbionts. Experientia 52, 716-722. doi: 10.1007/BF01925581

Blom, J., Albaum, S. P., Doppmeier, D., Pühler, A., Vorhölter, F.-J., Zakrzewski, M., et al. (2009). EDGAR: a software framework for the comparative analysis of prokaryotic genomes. BMC Bioinform. 10:154. doi: 10.1186/1471-2105-10-154

Bolger, A. M., Lohse, M., and Usadel, B. (2014). Trimmomatic: a flexible trimmer for Illumina sequence data. Bioinformatics 30, 2114-2120. doi: 10.1093/bioinformatics/btu170

Boyer, F., Fichant, G., Berthod, J., Vandenbrouck, Y., and Attree, I. (2009). Dissecting the bacterial type VI secretion system by a genome wide in silico analysis: what can be learned from available microbial genomic resources? $B M C$ Genomics 10:104. doi: 10.1186/1471-2164-10-104

Brettin, T., Davis, J. J., Disz, T., Edwards, R. A., Gerdes, S., Olsen, G. J., et al. (2015). RASTtk: a modular and extensible implementation of the RAST algorithm for building custom annotation pipelines and annotating batches of genomes. Sci. Rep. 5:8365. doi: 10.1038/srep08365

Brück, W. M., Sennett, S. H., Pomponi, S. A., Willenz, P., and McCarthy, P. J. (2008). Identification of the bacterial symbiont Entotheonella sp. in the mesohyl of the marine sponge Discodermia sp. ISME J. 2, 335-339. doi: 10.1038/ismej.2007.91

Burgsdorf, I., Slaby, B. M., Handley, K. M., Haber, M., Blom, J., Marshall, C. W., et al. (2015). Lifestyle evolution in cyanobacterial symbionts of sponges. MBio 6:e391-15. doi: 10.1128/mBio.00391-15

Capasso, C., and Supuran, C. T. (2015). An overview of the selectivity and efficiency of the bacterial carbonic anhydrase inhibitors. Curr. Med. Chem. 22, 2130-2139. doi: 10.2174/0929867321666141012174921 sequencing and assembling. GF sampled the sponges and extracted the DNA. All authors discussed and wrote the manuscript together.

\section{FUNDING}

The authors would like to thank the National Natural Science Foundation of China (NSFC) (U1301131, 41176127) and Minhang Leading Talent Project for providing research funding.

\section{SUPPLEMENTARY MATERIAL}

The Supplementary Material for this article can be found online at: http://journal.frontiersin.org/article/10.3389/fmicb. 2016.01333

Choudhury, J. D., Pramanik, A., Webster, N. S., Llewellyn, L. E., Gachhui, R., and Mukherjee, J. (2015). The pthogen of the great barrier reef sponge Rhopaloeides odorabile is a new strain of Pseudoalteromonas agarivorans containing abundant and diverse virulence-related genes. Mar. Biotechnol. 17, 463-478. doi: 10.1007/s10126-015-9627-y

Christin, P.-A., Arakaki, M., Osborne, C. P., Bräutigam, A., Sage, R. F., Hibberd, J. M., et al. (2014). Shared origins of a key enzyme during the evolution of C(4) and CAM metabolism. J. Exp. Bot. 65, 3609-3621. doi: 10.1093/jxb/eru087

Consortium, T. U. (2015). UniProt: a hub for protein information. Nucleic Acids Res. 43, D204-D212. doi: 10.1093/nar/gku989

Costa, T. R. D., Felisberto-Rodrigues, C., Meir, A., Prevost, M. S., Redzej, A., Trokter, M., et al. (2015). Secretion systems in Gram-negative bacteria: structural and mechanistic insights. Nat. Rev. Micro. 13, 343-359. doi: 10.1038/nrmicro3456

De Goeij, J. M., Moodley, L., Houtekamer, M., Carballeira, N. M., and Van Duyl, F. C. (2008). Tracing 13C-enriched dissolved and particulate organic carbon in the bacteria-containing coral reef sponge Halisarca caerulea: evidence for DOM feeding. Limnol. Oceanogr. 53, 1376-1386. doi: 10.4319/lo.2008.53.4.1376

De Goeij, J. M., Van Oevelen, D., Vermeij, M. J. A., Osinga, R., Middelburg, J. J., De Goeij, A. F. P. M., et al. (2013). Surviving in a marine desert: the sponge loop retains resources within coral reefs. Science 342, 108-110. doi: 10.1126/science. 1241981

Dodd, A. N., Borland, A. M., Haslam, R. P., Griffiths, H., and Maxwell, K. (2002). Crassulacean acid metabolism: plastic, fantastic. J. Exp. Bot. 53, 569-580. doi: 10.1093/jexbot/53.369.569

Edgar, R. C. (2004). MUSCLE: multiple sequence alignment with high accuracy and high throughput. Nucleic Acids Res. 32, 1792-1797. doi: 10.1093/nar/gkh340

Fan, L., Reynolds, D., Liu, M., Stark, M., Kjelleberg, S., Webster, N. S., et al. (2012). Functional equivalence and evolutionary convergence in complex communities of microbial sponge symbionts. Proc. Natl. Acad. Sci. U.S.A. 109, E1878-E1887. doi: 10.1073/pnas.1203287109

Freeman, M. F., Vagstad, A. L., and Piel, J. (2016). Polytheonamide biosynthesis showcasing the metabolic potential of sponge-associated uncultivated "Entotheonella" bacteria. Curr. Opin. Chem. Biol. 31, 8-14. doi: 10.1016/j.cbpa.2015.11.002

Friedrich, A. B., Fischer, I., Proksch, P., Hacker, J., and Hentschel, U. (2001). Temporal variation of the microbial community associated with the mediterranean sponge Aplysina aerophoba. FEMS Microbiol. Ecol. 38, 105-113. doi: 10.1016/S0168-6496(01)00185-4

Gao, Z. M., Wang, Y., Tian, R. M., Wong, Y. H., Batang, Z. B., Al-Suwailem, A. M., et al. (2014). Symbiotic adaptation drives genome streamlining of the cyanobacterial sponge symbiont "Candidatus Synechococcus spongiarum". MBio 5:e79-14. doi: 10.1128/mBio.00079-14 
Giovannoni, S. J., Cameron Thrash, J., and Temperton, B. (2014). Implications of streamlining theory for microbial ecology. ISME J. 8, 1553-1565. doi: 10.1038/ismej.2014.60

Hadas, E., Shpigel, M., and Ilan, M. (2009). Particulate organic matter as a food source for a coral reef sponge. J. Exp. Biol. 212, 3643-3650. doi: 10.1242/jeb.027953

He, L., Liu, F., Karuppiah, V., Ren, Y., and Li, Z. (2014). Comparisons of the fungal and protistan communities among different marine sponge holobionts by pyrosequencing. Microb. Ecol. 67, 951-961. doi: 10.1007/s00248-014-0393-6

Hentschel, U., Piel, J., Degnan, S. M., and Taylor, M. W. (2012). Genomic insights into the marine sponge microbiome. Nat. Rev. Microbiol. 10, 641-654. doi: $10.1038 /$ nrmicro2839

Hoffmann, F., Larsen, O., Thiel, V., Rapp, H. T., Pape, T., Michaelis, W., et al. (2005). An anaerobic world in sponges. Geomicrobiol. J. 22, 1-10. doi: 10.1080/01490450590922505

Hutchison, E. A., Miller, D. A., and Angert, E. R. (2014). Sporulation in bacteria: beyond the standard model. Microbiol. Spectr. 2. doi: 10.1128/microbiolspec.TBS-0013-2012.

Jourand, P., Giraud, E., Béna, G., Sy, A., Willems, A., Gillis, M., et al. (2004). Methylobacterium nodulans sp. nov., for a group of aerobic, facultatively methylotrophic, legume root-nodule-forming and nitrogen-fixing bacteria. Int. J. Syst. Evol. Microbiol. 54, 2269-2273. doi: 10.1099/ijs.0.02902-0

Kang, D. D., Froula, J., Egan, R., and Wang, Z. (2015). MetaBAT, an efficient tool for accurately reconstructing single genomes from complex microbial communities. PeerJ 3:e1165. doi: 10.7717/peerj.1165

Keren, R., Lavy, A., Mayzel, B., and Ilan, M. (2015). Culturable associated-bacteria of the sponge Theonella swinhoei show tolerance to high arsenic concentrations. Front. Microbiol. 6:154. doi: 10.3389/fmicb.2015.00154

Langdale, J. A. (2011). C4 cycles: past, present, and future research on C4 photosynthesis. Plant Cell 23, 3879-3892. doi: 10.1105/tpc.111.092098

Lavy, A., Keren, R., Haber, M., Schwartz, I., and Ilan, M. (2014). Implementing sponge physiological and genomic information to enhance the diversity of its culturable associated bacteria. FEMS Microbiol. Ecol. 87, 486-502. doi: 10.1111/1574-6941.12240

López-Pérez, M., Ghai, R., Leon, M. J., Rodríguez-Olmos, Á, Copa-Patiño, J. L., Soliveri, J., et al. (2013). Genomes of "Spiribacter", a streamlined, successful halophilic bacterium. BMC Genomics 14:787. doi: 10.1186/1471-2164-14-787

Luo, H., and Moran, M. A. (2015). How do divergent ecological strategies emerge among marine bacterioplankton lineages? Trends. Microbiol. 23, 577-584. doi: 10.1016/j.tim.2015.05.004

Maldonado, M., Ribes, M., and Van Duyl, F. C. (2012). Nutrient fluxes through sponges: biology, budgets, and ecological implications. Adv. Mar. Biol. 62, 113-167. doi: 10.1016/B978-0-12-394283-8.00003-5

Miyata, S. T., Bachmann, V., and Pukatzki, S. (2013). Type VI secretion system regulation as a consequence of evolutionary pressure. J. Med. Microbiol. 62, 663-676. doi: 10.1099/jmm.0.053983-0

Mohamed, N. M., Saito, K., Tal, Y., and Hill, R. T. (2010). Diversity of aerobic and anaerobic ammonia-oxidizing bacteria in marine sponges. ISME J. 4, 38-48. doi: 10.1038/ismej.2009.84

Namiki, T., Hachiya, T., Tanaka, H., and Sakakibara, Y. (2012). MetaVelvet: an extension of Velvet assembler to de novo metagenome assembly from short sequence reads. Nucleic Acids Res. 40:e155. doi: 10.1093/nar/gks678

Nguyen, M. T. H. D., Liu, M., and Thomas, T. (2014). Ankyrin-repeat proteins from sponge symbionts modulate amoebal phagocytosis. Mol. Ecol. 23, 1635-1645. doi: $10.1111 / \mathrm{mec} .12384$

Nicholson, W. L., Munakata, N., Horneck, G., Melosh, H. J., and Setlow, P. (2000). Resistance of Bacillus endospores to extreme terrestrial and extraterrestrial environments. Microbiol. Mol. Biol. Rev. 64, 548-572. doi: 10.1128/MMBR.64.3.548-572.2000

Piel, J., Hui, D., Wen, G., Butzke, D., Platzer, M., Fusetani, N., et al. (2004). Antitumor polyketide biosynthesis by an uncultivated bacterial symbiont of the marine sponge Theonella swinhoei. Proc. Natl. Acad. Sci. U.S.A. 101, 1622216227. doi: 10.1073/pnas.0405976101

Planet, P. J., Kachlany, S. C., Fine, D. H., DeSalle, R., and Figurski, D. H. (2003). The widespread colonization Island of Actinobacillus actinomycetemcomitans. Nat. Genet. 34, 193-198. doi: 10.1038/ng1154

Poehlein, A., Gottschalk, G., and Daniel, R. (2013). First insights into the genome of the Gram-gegative, endospore-forming organism Sporomusa ovata strain H1 DSM 2662. Genome Announc. 1:e00734-13. doi: 10.1128/genomeA. 00734-13
Pukatzki, S., Ma, A. T., Sturtevant, D., Krastins, B., Sarracino, D., Nelson, W. C., et al. (2006). Identification of a conserved bacterial protein secretion system in Vibrio cholerae using the Dictyostelium host model system. Proc. Natl. Acad. Sci. U.S.A. 103, 1528-1533. doi: 10.1073/pnas.0510322103

Reveillaud, J., Maignien, L., Eren, A. M., Huber, J. A., Apprill, A., Sogin, M. L., et al. (2014). Host-specificity among abundant and rare taxa in the sponge microbiome. ISME J. 8, 1198-1209. doi: 10.1038/ismej.2013.227

Richter, M., and Rosselló-Móra, R. (2009). Shifting the genomic gold standard for the prokaryotic species definition. Proc. Natl. Acad. Sci. U.S.A. 106, 1912619131. doi: 10.1073/pnas.0906412106

Richter, M., Rossello-Mora, R., Oliver Glockner, F., and Peplies, J. (2016). JSpeciesWS: a web server for prokaryotic species circumscription based on pairwise genome comparison. Bioinformatics. 32, 929-931. doi: 10.1093/bioinformatics/btv681

Schmidt, E. W., Obraztsova, A. Y., Davidson, S. K., Faulkner, D. J., and Haygood, M. G. (2000). Identification of the antifungal peptidecontaining symbiont of the marine sponge Theonella swinhoei as a novel $\delta$-proteobacterium, “Candidatus Entotheonella palauensis”. Mar. Biol. 136, 969977. doi: $10.1007 /$ s002270000273

Schmitt, S., Tsai, P., Bell, J., Fromont, J., Ilan, M., Lindquist, N., et al. (2012). Assessing the complex sponge microbiota: core, variable and speciesspecific bacterial communities in marine sponges. ISME J. 6, 564-576. doi: 10.1038/ismej.2011.116

Siegl, A., Kamke, J., Hochmuth, T., Piel, J., Richter, M., Liang, C., et al. (2011). Single-cell genomics reveals the lifestyle of Poribacteria, a candidate phylum symbiotically associated with marine sponges. ISME J. 5, 61-70. doi: 10.1038/ismej.2010.95

Tamura, K., Stecher, G., Peterson, D., Filipski, A., and Kumar, S. (2013). MEGA6: molecular evolutionary genetics analysis version 6.0. Mol. Biol. Evol. 30, 27252729. doi: 10.1093/molbev/mst197

Taylor, M. W., Hill, R. T., Piel, J., Thacker, R. W., and Hentschel, U. (2007). Soaking it up: the complex lives of marine sponges and their microbial associates. ISME J. 1, 187-190. doi: 10.1038/ismej.2007.32

Tian, R.-M., Sun, J., Cai, L., Zhang, W.-P., Zhou, G.-W., Qiu, J.-W., et al. (2016). The deep-sea glass sponge Lophophysema eversa harbors potential symbionts responsible for the nutrient conversions of carbon, nitrogen and sulfur. Environ. Microbiol. doi: 10.1111/1462-2920.13161 [Epub ahead of print].

Tomich, M., Planet, P. J., and Figurski, D. H. (2007). The tad locus: postcards from the widespread colonization island. Nat. Rev. Microbiol. 5, 363-375. doi: 10.1038/nrmicro1636

Ueoka, R., Uria, A. R., Reiter, S., Mori, T., Karbaum, P., Peters, E. E., et al. (2015). Metabolic and evolutionary origin of actin-binding polyketides from diverse organisms. Nat. Chem. Biol. 11, 705-712. doi: 10.1038/nchembio.1870

Wattam, A. R., Abraham, D., Dalay, O., Disz, T. L., Driscoll, T., Gabbard, J. L., et al. (2014). PATRIC, the bacterial bioinformatics database and analysis resource. Nucleic Acids Res. 42, D581-D591. doi: 10.1093/nar/gkt1099

Webster, N. S., Taylor, M. W., Behnam, F., Lücker, S., Rattei, T., Whalan, S., et al. (2010). Deep sequencing reveals exceptional diversity and modes of transmission for bacterial sponge symbionts. Environ. Microbiol. 12, 20702082. doi: 10.1111/j.1462-2920.2009.02065.x

Webster, N. S., and Thomas, T. (2016). The sponge hologenome. MBio 7:e135-16. doi: $10.1128 / \mathrm{mBio} .00135-16$

Wilson, M. C., Mori, T., Rückert, C., Uria, A. R., Helf, M. J., Takada, K., et al. (2014). An environmental bacterial taxon with a large and distinct metabolic repertoire. Nature 506, 58-62. doi: 10.1038/nature 12959

Yahel, G., Sharp, J. H., Marie, D., Hase, A., and Genin, A. (2003). In situ feeding and element removal in the symbiont-bearing sponge Theonella swinhoei: bulk DOC is the major source for carbon. Limnol. Oceanogr. 48, 141-149. doi: 10.1038 /nature12959

Conflict of Interest Statement: The authors declare that the research was conducted in the absence of any commercial or financial relationships that could be construed as a potential conflict of interest.

Copyright (c) $2016 \mathrm{Liu}, \mathrm{Li}$, Feng and Li. This is an open-access article distributed under the terms of the Creative Commons Attribution License (CC BY). The use, distribution or reproduction in other forums is permitted, provided the original author(s) or licensor are credited and that the original publication in this journal is cited, in accordance with accepted academic practice. No use, distribution or reproduction is permitted which does not comply with these terms. 